\title{
Phenyl Substituted Porphyrins. Part 4. Acylation of Hydroxyphenylporphyrins
}

\author{
Mariya A. Salnikova, Tatiana V. Lubimova, Aleksey V. Glazynov, Sergei A. Syrbu, ${ }^{@}$ \\ Alexander S. Semeikin and Oscar I. Koifman
}

Dedicated to Academician Irina P. Beletskaya on the occasion of her birthday

Ivanovo State University of Chemistry and Technology, 153000 Ivanovo, Russia

${ }^{\circledR}$ Corresponding author E-mail: syrbu@isuct.ru

Acylation of hydroxyphenylporphyrins by carboxylic acids in the presence of carbodiimides was studied and the optimal reaction conditions were determined. Some new esters of various hydroxyphenylporphyrins were synthesized.

Keywords: Porphyrins, acylation, DCC, EDAC, 4-dimethylaminopyridine (DMAP).

\section{Фенилзамещённые порфирины. 4. Ацилирование гидроксифенилпорфиринов}

\author{
М. А. Сальникова, Т. В. Аюбимова, А. В. Глазунов, С. А. Сырбу,
}

А. С. Семейкин, О. И. Койфрман

Посвящается акаgемику И. П. Белецкой по случаю её юбилея

Ивановский государственный химико-технологический университет, 153000 Иваново, Россия

${ }^{\circledR}$ E-mail: syrbu@isuct.ru

\begin{abstract}
Изучены условия ацилирования гидроксифенилпорфиринов карбоновыми кислотами в присутствии карбодиимидов. Найдены оптимальные условия проведения реакиии и синтезирован ряд сложных эфиров гидроксифенилпорфиринов различной структурь.
\end{abstract}

Ключевые слова: Порфирины, ацилирование, дициклогексилкарбодиимид (ДЦК), 1-(3-диметиламинопропил)3-этилкарбодиимид гидрохлорид (ДЭКГ), 4-диметиламинопиридин (ДМАП).

\section{Introduction}

Porphyrins, having phenyl rings in meso-positions, are mostly known and available, since they can be easily obtained in high yields by condensation of substituted benzaldehydes with pyrrole or its linear derivatives (dipyrrolylmethanes or biladiens). Thus, these porphyrins are widely used in biological investigations, as catalysts of various processes and as medical preparations ..$^{[1-6]}$

Active groups in phenyl rings of phenyl substituted porphyrins (i.e., oxy or amino groups) may be rather easily modified. This allows to obtain the porphyrins with the substituents, which are able to interact with the inner porphyrin reaction center containing metal atom, to bind the porphyrins to various substrates and to favour the formation of nanostructures .

The most simple modification of oxy groups in the phenyl rings may be performed by their alkylation or acylation. Alkylation is realized by interaction of oxy groups with halogen derivatives in polar solvents in the presence of bases (usually DMF with potassium carbonate) and is rather widely studied. ${ }^{[7-10]}$ At the same time acylation may be performed by different carboxylic acid derivatives (anhydrides or halogenanhydrides $)^{[1-14]}$ or by carboxylic acids themselves in the presence of dicyclohexylcarbodiimide (DCC). ${ }^{[15-18]}$ The latter is more preferred, since the active derivatives of carboxylic acids can not be always obtained, and, moreover, the method with DCC allows to exclude the intermediate stages . 
In this paper we describe the convenient and general method elaborated for acylation of hydroxyphenylporphyrins, that allows modifying of the initial phenylporphyrins with formation of various structures .

\section{Results and Discussion}

As the initial hydroxyphenylporphyrins we have used the relatively easily available isomeric 5-hydroxyphenyl10,15,20-tiphenylporphines $\mathbf{1}$, which were obtained by "mixed aldehyde" condensation of hydroxybenzaldehydes $\mathbf{6}$ and benzaldehyde 7 with pyrrole $\mathbf{5}$ in binary mixture xylenetrifluoriacetic acid (Scheme 1). The attempts to synthesize these pophyrins by Lindsey method ${ }^{[19-24]}$ did not lead to the desired result. Nevertheless, isomeric porphyrins 1 were obtained by two-stage scheme through 5-methoxyphenyl10,15,20-triphenylporphyrins $\mathbf{1 0}$ with following demethylation of the porphyrin mixture by boron tribromide ${ }^{[25]}$ and its chromatographic separation. It should be noted, that this method does not has any advantages over one-stage method, but allows the preparation of porphyrins containg more than one hydroxy group. Isolation of such porphyrins from the mixture obtained by one-stage procedure is very troblesome .

meso-Tetrakis(hydroxyphenyl)porphyrins $\mathbf{2}$ are formed with low yield by direct condensation reaction of hydroxybenzaldehydes $\mathbf{6}$ with pyrrole $\mathbf{5}$, thus they were synthesized by demethylation of more available tetrakis(methoxyphenyl)porphyrins 11 (Scheme 2). ${ }^{[26,27]}$

Isomeric 5-hydroxyphenyl-2,3,7,8,12,18-hexamethyl13,18-di- $n$-amylporphyrins 3 were synthesized by condensation of 4,4'-dimethyl-3,3'-di- $n$-amyldipyrrolylmethane $\mathbf{1 2}^{\text {[28] }}$ with 2-formyl-3,4-dimethylpyrrole $\mathbf{1 3}^{[28]}$ in the presence of hydrobromic acid in alcohol leading to the corresponding biladiene $\mathbf{1 4}$ and its following interaction without isolation with hydroxybenzaldehydes $\mathbf{6}$, similarly to ${ }^{[29,30]}$ (Scheme 3).

11-Bromoundecanoic acid $15\left(\mathrm{R}=\left(\mathrm{CH}_{2}\right)_{10} \mathrm{Br}\right)$ was used in the model reactions as acylation agent in the synthesis of porphyrins, containing the acyl group with terminal bromine atom, which later can be used in the nucleophilic substitution reactions .

In the process of our study it was established, thatacylation of 1 (para) by chloroanhydride of 11-bromoundecanoic acid in pyridine similarly to ${ }^{[13]}$ does not procceds, as well as in the case of acid in the presence of $N, N^{\prime}$-carbonyldiimidazole (CDI) as an activating agent. Therefore the acylation reaction in the presence of carbodiimide $\mathbf{1 6}$ derivatives as activator was studied (Scheme 4). There are two known effective carbodiimides: $N, N$ '-dicyclohexylcarbodiimide $\left(\mathbf{1 6}, \mathrm{R}^{\prime}=\mathrm{R}\right.$ " $=$ $\left.-\mathrm{C}_{6} \mathrm{H}_{11}\right)(\mathrm{DCC})$ and 1-(3-dimehylaminopropyl)-3-ethylcarbodiimide hydrochloride $\left(\mathbf{1 6}, \mathrm{R}^{\prime}=-\left(\mathrm{CH}_{2}\right)_{3} \mathrm{NH}^{+}\left(\mathrm{CH}_{3}\right)_{2} \cdot \mathrm{Cl}^{-} ; \mathrm{R}^{\prime}\right.$ '
4<smiles></smiles>

5<smiles>O=Cc1cccc(O)c1</smiles>

6<smiles>O=Cc1ccccc1</smiles>

$\underset{\text { xylene }}{\stackrel{\mathrm{H}^{+} ; \mathrm{O}_{2}}{\longrightarrow}}$ 7<smiles>Oc1ccc(C(c2ccc(-c3ccccc3)[nH]2)=c2ccc(=C(c3ccccc3)c3ccc(-c4ccccc4)[nH]3)[nH]2)cc1</smiles>

1

$\mathrm{BBr}_{3} \uparrow$<smiles>CCOC1CC1</smiles>

$\underset{\text { xylene }}{\stackrel{\mathrm{H}^{+} ; \mathrm{O}_{2}}{\longrightarrow}}$<smiles>COc1ccc(C(c2ccc([C+](c3ccccc3)c3ccccc3)[nH]2)=c2ccc(=c3ccc(=C4C=CC(c5ccccc5)=N4)[nH]3)[nH]2)cc1</smiles>

10

Scheme 1.

4

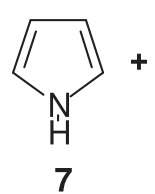

7

4<smiles>COc1ccc(C=O)cc1</smiles><smiles>COc1cccc(C=O)c1</smiles>

8<smiles></smiles>

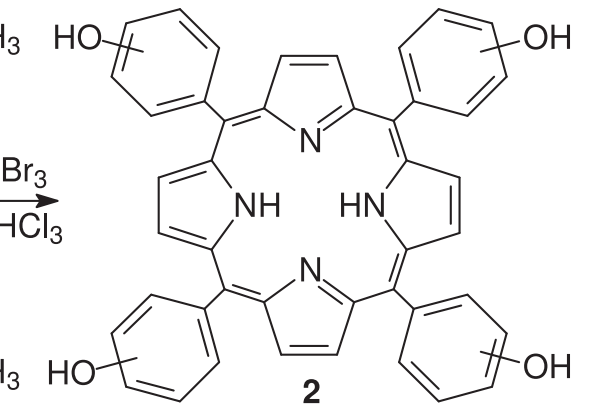

Scheme 2. 
$\left.=\mathrm{C}_{2} \mathrm{H}_{5}\right)($ EDAC). Each of them has some advantages and disadvantages. We have established, that for the completion of the acylation reaction slightly larger quantity of DCC is required than DECC. On the other side, DCC is cheaper and more available as compared to DECC. At the equal molar ratio porphyrin:reagent the yield in the case of DECC is slightly higher $-71.6 \%$ vs. $66.5 \%$. However, it should be taken into account that in the case of DCC the second reaction product (dicyclohexylurea 17, R' $=\mathrm{R}^{\prime \prime}=\mathrm{C}_{6} \mathrm{H}_{11}$ ) might contaminate the target product during the chromatographic purification.

Dichloromethane was chosen as a solvent for the reaction, since practically all the porphyrins and other reagents are well soluble in it, and, moreover, the reaction mixture in this case can be directly chromatographed without additional manipulations. The use of other solvents, THF or DMF, also leads to the positive results and may be applied in acylation of tetrakis(hydroxyphenyl)porphyrins, which are low-soluble in dichloromethane; but in this case the acylation proceeds considerably slower and, besides, these solvents require thorough laborious drying.

4-Dimethylaminopyridine (DMAP) was used as a catalyst according to the literature. ${ }^{[15-18]}$ The acylation does not take place without it and its quantity plays a definite role. We have established, that its optimal molar ratio to porphyrin is about 0.5 .

According to Scheme 4 it is obvious, that the quantity of carbodiimide should be no less than one equivalent to one equivalent of acylated hydroxy group. We have observed that quantity of carbodiimide must be 1.5-2 times higher, perhaps due to the presence of residual water in the solvent.

Acylation was carried out sequentially by interaction of carbodiimide with an acid upon cooling to $0{ }^{\circ} \mathrm{C}$ for 1.5 hours, and then at room temperature. The reaction process was controlled by thin layer chromatography. It turns out, that the reaction completion requires about 3 hours, but the reaction time is increased in the case of ortho-hydroxyphenylporphyrins and tetrakis(hydroxyphenyl)porphyrins or when using low active carboxylic acids are used for acylation. The reaction process without preliminary cooling decreases the yield of acylated porphyrin approximately in two times.
If the reaction is carried out under reflux, the yield of the acylated porphyrin is lowered because of the formation of by-products.

The yields of the acylated porphyrins are increased when going from meso-phenyl substituted hydroxyphenylporphyrins $\mathbf{1}$ to $\beta$-alkyl substituted porphyrins $\mathbf{2}$, the reaction time being increased. The position of hydroxy group in phenyl ring only slightly influences on the yield of acylated porphyrins.

It is interesting that tetrakis (hydroxyphenyl)porphyrins are not fully acylated and form a mixture of acylated species. The relative yield of tetraacylated porphyrin is increased neither with elongation of the reaction time nor at higher molar ratio of acylating mixture to the porphyrin.

Change of aliphatic carboxylic acid on the aromatic one leads to the significant increase of the acylation reaction yield, perhaps because of increasing stability of the obtained porphyrin ester to hydrolysis. Analogously, one can explain the yield decreasing and reaction rime elongation in acylation of hydroxyphenylporphyrins $\mathbf{1}$ (para) and $\mathbf{2}$ (para) by $N$-BOC protected aminoacids.

In the ${ }^{1} \mathrm{H}$ NMR spectra of the synthesized porphyrins 4 (ortho, $\mathrm{R}=\left(\mathrm{CH}_{2}\right)_{10} \mathrm{Br}$ ) and $\mathbf{5}$ (ortho, $\mathrm{R}=\left(\mathrm{CH}_{2}\right)_{10} \mathrm{Br}$ ) the slight downfield shift and the full splitting of the methylene signals of the acyl unit are observed that is caused by the ring current of porphyrin fragment.

\section{Experimental}

UV-vis spectra were recorded on a scanning spectrometer SPEC SSP-715 in chloroform, IR spectra on a Avatar 360 FT-IR spectrphtometer in $\mathrm{KBr}$ tablets and ${ }^{1} \mathrm{H}$ NMR spectra on a Bruker $500 \mathrm{MHz}$ spectrometer in $\mathrm{CDCl}_{3}$ (internal standard TMS). Thin layer chromatography (TLC) was made on Silufol ${ }^{\circledR}$ plates.

5-(4'-Hydroxyphenyl)-10,15,20-triphenylporphine 1 (para).

a) To a boiling solution of trifluoroacetic acid $(4.0 \mathrm{ml}$, $53.8 \mathrm{mmol}, \mathrm{ca} .2 \%$ ) in $300 \mathrm{ml}$ of $p$-xylene the solution of 4-hydroxybenzaldehyde $(2.2 \mathrm{~g}, 18.5 \mathrm{mmol})$, benzaldehyde $(5.5 \mathrm{ml}$, $54.2 \mathrm{mmol})$ and pyrrole $(5.0 \mathrm{ml}, 72.2 \mathrm{mmol})$ in $50 \mathrm{ml}$ of $p$-xylene was smoothly added under argon. The mixture was refluxed for $0.5 \mathrm{~h}$ under Ar and then $1 \mathrm{~h}$ in the presence of air, the solvent was

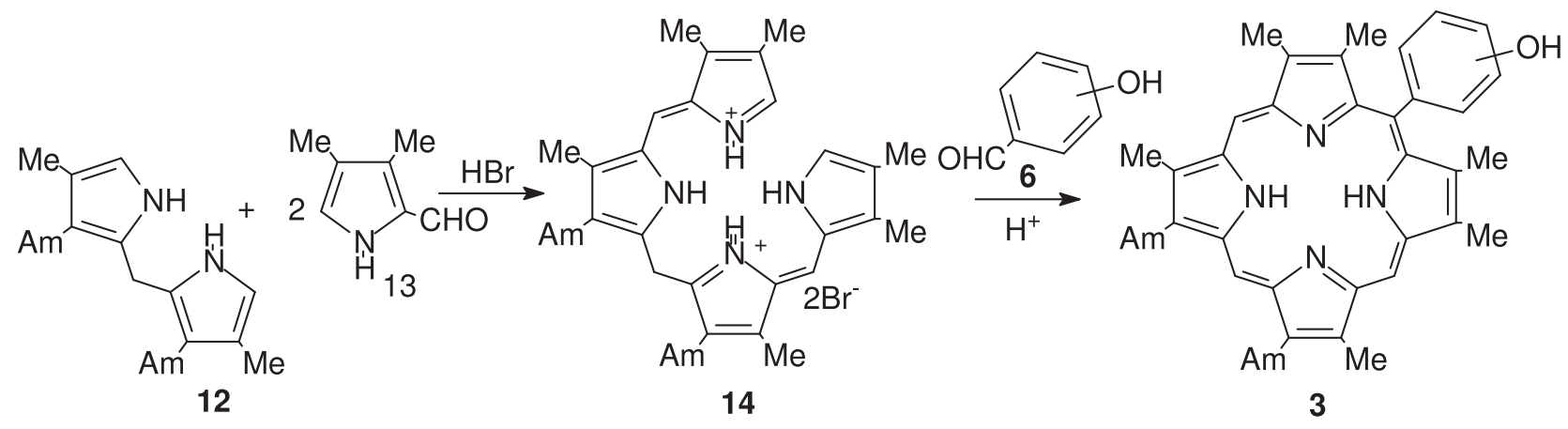

Scheme 3 .

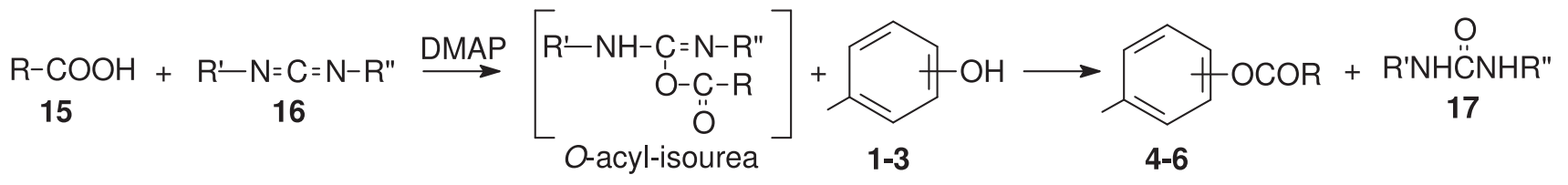

Scheme 4. 
distilled off with water vapour, the residue was filtered, washed with water and dried at $70{ }^{\circ} \mathrm{C}$. The mixture was dissolved in chloroform and chromatographed on $\mathrm{Al}_{2} \mathrm{O}_{3}$ (II Brockman degree). The first fraction was eluted by chloroform, eluate was evaporated and the porphyrin was precipitated by methanol. Yield $1.1 \mathrm{~g}(10 \%)$. The second fraction was rinsed by chloroform-methanol mixture, evaporated to dryness, dissolved in $\mathrm{CH}_{2} \mathrm{Cl}_{2}$ and chromatographed on silica collecting the second fraction. The eluate was evaporated and the porphyrin was precipitated by petroleum ether. Yield 1.05 $\mathrm{g}(9.2 \%)$.

b) To a boiling solution of trifluoroacetic acid $(4.0 \mathrm{ml}, 53.8$ mmol, ca. $2 \%$ ) in $300 \mathrm{ml}$ of $p$-xylene the solution of anisaldehyde $(2.2 \mathrm{ml}, 18.05 \mathrm{mmol})$, benzaldehyde $(5.5 \mathrm{ml}, 54.2 \mathrm{mmol})$ and pyrrole $(5.0 \mathrm{ml}, 72.2 \mathrm{mmol})$ in $20 \mathrm{ml}$ of $p$-xylene was smoothly added under argon. The mixture was refluxed for $0.5 \mathrm{~h}$ under Ar and then $1 \mathrm{~h}$ in the air, the solvent was distilled off with water vapour, and the residue was filtered, washed with water and dried at $70{ }^{\circ} \mathrm{C}$. The mixture was dissolved in chloroform and chromatographed on $\mathrm{Al}_{2} \mathrm{O}_{3}$ (II Brockmann degree). The first fraction was eluted by chloroform, the eluate was evaporated and the mixture of porphyrins was precipitated by methanol. Yield of the mixture of methoxyphenylporphyrins and tetraphenylporphine $4.4 \mathrm{~g}$. The precipitate obtained was dissolved with stirring in $150 \mathrm{ml}$ of dried dichloromethane (freed from alcohol by passing through $\mathrm{Al}_{2} \mathrm{O}_{3}$ of I Brockmann degree). To the obtained solution boron tribromide $(4.5 \mathrm{ml}, 47.6 \mathrm{ml})$ was smoothly added at room temperature. The mixture was stirred for 2 hours, mixed with methanol ( $4.5 \mathrm{ml}, 47.6 \mathrm{mmol})$ and neutralized with aqueous ammonia solution $(15 \mathrm{ml})$ till the colour change from green to reddish-brown. The solution was evaporated to dryness, washed with water from inorganic salts, and dried at $70{ }^{\circ} \mathrm{C}$. The precipitate was dissolved in $\mathrm{CH}_{2} \mathrm{Cl}_{2}$ and chromatographed on silica by dichloromethane. The first fraction containing tetraphenylporphine was collected, evaporated and precipitated with methanol. Yield $1.3 \mathrm{~g}(12 \%)$. Then the second fraction of the product was collected, evaporated and precipitated with petroleum ether. Yield $0.82 \mathrm{~g}(7 \%)$.

$R_{\mathrm{f}}=0.61$ (benzene-methanol, 10:1). UV-vis $\lambda \mathrm{nm}(\lg \varepsilon)$ : 649 (3.90), 592 (3.86), 552 (4.03), 516 (4.1), 419 (5.93). IR $v \mathrm{~cm}^{-1}$ : $3600(\mathrm{vOH}), 1350(\delta \mathrm{OH}) .{ }^{1} \mathrm{H}$ NMR $\delta$ ppm: $8.89 \mathrm{~m}(8 \mathrm{H}, \beta-\mathrm{H}), 8.25$ $\mathrm{d}(6 \mathrm{H}, o-\mathrm{H}-\mathrm{Ph}), 8.07 \mathrm{~d}\left(2 \mathrm{H}, 2^{\prime}, 6\right.$ '-H-Ar), $7.86 \mathrm{~m}(9 \mathrm{H}, m, p-\mathrm{H}-\mathrm{Ph})$, $7.14 \mathrm{~d}(2 \mathrm{H}, 3$ ', 5'-H-Ar), -2.74b s (2H, NH).

5-(3'-Hydroxyphenyl)-10,15,20-triphenylporphine, 1 (meta) was obtained analogously to method $a$. Yield $11 \% . R_{\mathrm{f}}=0.65$ (benzenemethanol, 10:1). UV-vis $\lambda_{\max } \mathrm{nm}$ (lge): 647 (3.72), 591 (3.85), 550 (3.95), 515 (4.33), 419 (5.85). IR $v \mathrm{~cm}^{-1}: 3600(\mathrm{vOH}), 1370(\delta \mathrm{OH}) .{ }^{1} \mathrm{H}$ NMR $\delta$ ppm: $8.91 \mathrm{~d}(2 \mathrm{H}, \beta-\mathrm{H}), 8.6 \mathrm{~m}(6 \mathrm{H}, \beta-\mathrm{H}), 8.23 \mathrm{~d}(6 \mathrm{H}, o-\mathrm{H}-\mathrm{Ph})$, $7.83 \mathrm{~d}\left(1 \mathrm{H}, 6\right.$ '-H-Ar), $7.78 \mathrm{~m}(9 \mathrm{H}, m, p-\mathrm{H}-\mathrm{Ph}), 7.76 \mathrm{~s}\left(1 \mathrm{H}, 2^{\prime}-\mathrm{H}-\mathrm{Ar}\right)$, 7.65 t (1H, 5'-H-Ar), 7.35 d (1H, 4'-H-Ar), -2.76 bs (2H, NH).

5-(2'-Hydroxyphenyl)-10,15,20-triphenylporphine, 1 (ortho) was obtained analogiously to method $a$. Yield 5.3\%. $R_{\mathrm{f}}=0.82$ (benzene-methanol, 10:1). UV-vis $\lambda_{\text {max. }}$ nm (lge): 649 (3.77), 589 (3.81), 549 (3.89), 515 (4.29), 419 (5.85). IR $v \mathrm{~cm}^{-1}: 3540(\mathrm{vOH})$, $1360(\delta \mathrm{OH}) .{ }^{1} \mathrm{H}$ NMR $\delta$ ppm: $8.89 \mathrm{~d}(2 \mathrm{H}, \beta-\mathrm{H}), 8.88 \mathrm{~m}(6 \mathrm{H}, \beta-\mathrm{H})$, $8.22 \mathrm{~m}(6 \mathrm{H}, o-\mathrm{H}-\mathrm{Ph}), 8.01 \mathrm{~d}\left(1 \mathrm{H}, 6^{\prime}-\mathrm{H}-\mathrm{Ar}\right), 7.74 \mathrm{t}\left(1 \mathrm{H}, 5^{\prime}-\mathrm{H}-\mathrm{Ar}\right)$, $7.70 \mathrm{~m}(9 \mathrm{H}, m, p-\mathrm{H}-\mathrm{Ph}), 7.52 \mathrm{t}$ (1H, 4'-H-Ar), $7.36 \mathrm{~d}(1 \mathrm{H}, 3$ '-H-Ar), 5.02 bs $(1 \mathrm{H}, \mathrm{OH}),-2.71$ bs $(2 \mathrm{H}, \mathrm{NH})$.

5-(4'-Hydroxyphenyl)-2,3, 7,8,12,18-hexamethyl-13,17-di-namylporphine, 3 (para). To a stirred solution of 4,4'-dimethyl-3,3'diamyldipyrrolylmethane $(1.33 \mathrm{~g}, 4.32 \mathrm{mmol})$ and 2-formyl-3,4dimethylpyrrole $(1.1 \mathrm{~g}, 8.64 \mathrm{mmol})$ in methanol $(100 \mathrm{ml})$ conc. $\mathrm{HBr}$ $(2 \mathrm{ml}, 16.6 \mathrm{mmol}$ ) was added at room temperature (the precipitate of biladiene was formed). After 1 hour 4-oxybenzaldehyde (4 g, $32.8 \mathrm{mmol}, 8$-fold excess) was added, and the mixture obtained was refluxed for 4 hours. Then aqueous ammonia $(4 \mathrm{ml})$ in 100 $\mathrm{ml}$ of water was smoothly added and the mixture was cooled. The precipitate was filtered, washed with water and dried at $70{ }^{\circ} \mathrm{C}$. The precipitate was dissolved in dichloromethane and chromatographed on $\mathrm{Al}_{2} \mathrm{O}_{3}$ (II degree) first by $\mathrm{CH}_{2} \mathrm{Cl}_{2}$ to remove the traces of the corresponding corrole. The second fraction containing porphyrin was eluted by $\mathrm{CH}_{2} \mathrm{Cl}_{2}-\mathrm{CH}_{3} \mathrm{OH}$ mixture. Eluate was evaporated and the pophyrin was precipitated by methanol, filtered off, washed with methanol and dried at $70{ }^{\circ} \mathrm{C}$. Yield $1.24 \mathrm{~g}(46 \%) . R_{\mathrm{f}}=0.62$ (benzene-methanol, 10:1). UV-vis $\lambda_{\text {max }} \mathrm{nm}(\lg \varepsilon): 624$ (3.51), 571 (3.88), 537 (3.90), 503 (4.21), 404 (5.29). IR $v \mathrm{~cm}^{-1}$ : 3431 (vOH), $1399(\delta \mathrm{OH}) .{ }^{1} \mathrm{H}$ NMR $\delta \mathrm{ppm}: 10.13 \mathrm{~s}(2 \mathrm{H}, 10,20-\mathrm{H}), 9.93 \mathrm{~s}(1 \mathrm{H}$, 15-H), 7.86 d (2H, 2',6'-H-Ar), 7.17 d (2H, 3', 5'-H-Ar), 4.04 t (4H, $\left.\mathrm{CH}_{2}-\mathrm{Am}\right), 3.63 \mathrm{~s}\left(6 \mathrm{H}, 12,18-\mathrm{CH}_{3}\right), 3.53 \mathrm{~s}\left(6 \mathrm{H}, 2,8-\mathrm{CH}_{3}\right), 2.52 \mathrm{~s}$ $\left(6 \mathrm{H}, 3,7-\mathrm{CH}_{3}\right), 2.31 \mathrm{qv}\left(4 \mathrm{H}, \mathrm{CH}_{2}-\mathrm{Am}\right), 1.73 \mathrm{qv}\left(4 \mathrm{H}, \mathrm{CH}_{2}-\mathrm{Am}\right), 1.56$ $\mathrm{sc}\left(4 \mathrm{H}, \mathrm{CH}_{2}-\mathrm{Am}\right), 0.99 \mathrm{t}\left(6 \mathrm{H}, \mathrm{CH}_{3}-\mathrm{Am}\right)$.

5-(3'-Hydroxyphenyl)-2,3,7,8,12,18-hexamethyl-13,17-di-namylporphine, 3 (meta). To a stirred solution of 4,4'-dimethyl-3,3'diamyldipyrrolylmethane (1.2 g, $3.82 \mathrm{mmol})$ and 2-formyl-3,4dimethylpyrrole $(0.94 \mathrm{~g}, 5.23 \mathrm{mmol})$ in butanol $(100 \mathrm{ml})$ conc. $\mathrm{HBr}$ $(2 \mathrm{ml}, 16.6 \mathrm{mmol}$ ) was added at room temperature (the precipitate of biladiene was formed). After 1 hour 4-hydroxybenzaldehyde (2 g, $16.4 \mathrm{mmol}$, 4-fold excess) was added, and the mixture obtained was refluxed for 4 hours. Then conc. ammonia solution $(4 \mathrm{ml})$ was smoothly added and the mixture was cooled. The precipitate was filtered, washed with methanol and dried at $70{ }^{\circ} \mathrm{C}$. The porphyrin was dissolved in dichloromethane and chromatographed on silica. Eluate was evaporated and the pophyrin was precipitated by methanol. Yield $0.92 \mathrm{~g}(38.4 \%) . R_{\mathrm{f}}=0.66$ (benzene-methanol, 10:1). UV-vis $\lambda_{\max } \mathrm{nm}$ (lge): 623 (3.56), 570 (3.91), 537 (3.94), 503 (4.23), 404 (5.32). IR v $\mathrm{cm}^{-1}: 3540(\mathrm{vOH}), 1396(\delta \mathrm{OH}) .{ }^{1} \mathrm{H}$ NMR $\delta$ ppm: $10.14 \mathrm{~s}(2 \mathrm{H}, 10,20-\mathrm{H})$, $9.89 \mathrm{~s}(1 \mathrm{H}, 15-\mathrm{H}), 7.45 \mathrm{~m}$ (2H, 2',6'-H-Ar), $7.06 \mathrm{~m}$ (1H, 5'-H-Ar), 6.87 m (1H, 4'-H-Ar), 3.97 t (4H, $\left.\mathrm{CH}_{2}-\mathrm{Am}\right), 3.60 \mathrm{~s}\left(6 \mathrm{H}, 12,18-\mathrm{CH}_{3}\right), 3.51$ $\mathrm{s}\left(6 \mathrm{H}, 2,8-\mathrm{CH}_{3}\right), 2.35 \mathrm{~s}\left(6 \mathrm{H}, 3,7-\mathrm{CH}_{3}\right), 2.29 \mathrm{qv}\left(4 \mathrm{H}, \mathrm{CH}_{2}-\mathrm{Am}\right), 1.79 \mathrm{qv}$ (4H, $\left.\mathrm{CH}_{2}-\mathrm{Am}\right), 1.54 \mathrm{sc}\left(4 \mathrm{H}, \mathrm{CH}_{2}-\mathrm{Am}\right), 0.98 \mathrm{t}\left(6 \mathrm{H}, \mathrm{CH}_{3}-\mathrm{Am}\right)$.

5-(2'-Hydroxyphenyl)-2,3, 7,8,12,18-hexamethyl-13,17-di-namylporphine, 3 (ortho). To a stirred solution of 4,4'-dimethyl-3,3'diamyldipyrrolylmethane $(0.96 \mathrm{~g}, 3.05 \mathrm{mmol})$ and 2-formyl-3,4dimethylpyrrole $(0.75 \mathrm{~g}, 6.09 \mathrm{mmol})$ in butanol $(100 \mathrm{ml})$ conc. $\mathrm{HBr}$ $(2 \mathrm{ml}, 16.6 \mathrm{mmol}$ ) was added at room temperature (the precipitate of biladiene was formed). After 1 hour salicylaldehyde ( $2 \mathrm{ml}, 19.1$ mmol, 6-fold excess) was added, and the mixture obtained was refluxed for 4 hours. Then $p$-benzoquinone $(0.66 \mathrm{~g}, 6.1 \mathrm{mmol})$ was added and the mixture was stirred for $10 \mathrm{~min}$. Butanol was distilled off with water vapour, the residue was filtered off, washed with water and dried at $70{ }^{\circ} \mathrm{C}$. The precipitate was dissolved in $\mathrm{CH}_{2} \mathrm{Cl}_{2}$ and chromatographed on $\mathrm{Al}_{2} \mathrm{O}_{3}$ (II degree). The eluate was thoroughly evaporated, dissolved in benzene and chromatographed once again on silica by benzene. The eluate was evaporated and pophyrin was precipitated by methanol, filtered off, washed with methanol and dried at $70{ }^{\circ} \mathrm{C}$. Yield $0.66 \mathrm{~g}(34.5 \%) . R_{\mathrm{f}}=0.83$ (benzene-methanol, 10:1). UV-vis $\lambda_{\max } \mathrm{nm}(\lg \varepsilon): 623$ (3.64), 572 (3.88), 537 (3.95), 503 (4.21), $403(5.28)$. IR $v \mathrm{~cm}^{-1}: 3421(\mathrm{vOH}), 1106(\delta \mathrm{OH}) .{ }^{1} \mathrm{H}$ NMR $\delta$ ppm: $10.21 \mathrm{~s}(2 \mathrm{H}, 10,20-\mathrm{H}), 10.02 \mathrm{~s}(1 \mathrm{H}, 15-\mathrm{H}), 7.76 \mathrm{t}+\mathrm{d}(2 \mathrm{H}$, 4'+6'-H-Ar), 7.40 d (1H, 3'-H-Ar), 7.36 t (1H, 5'-H-Ar), $4.06 \mathrm{t}$ (4H, $\left.\mathrm{CH}_{2}-\mathrm{Am}\right), 3.66 \mathrm{~s}\left(6 \mathrm{H}, 12,18-\mathrm{CH}_{3}\right), 3.58 \mathrm{~s}\left(6 \mathrm{H}, 2,8-\mathrm{CH}_{3}\right), 2.64$ s $\left(6 \mathrm{H}, 3,7-\mathrm{CH}_{3}\right), 2.33$ qv $\left(4 \mathrm{H}, \mathrm{CH}_{2}-\mathrm{Am}\right), 1.76 \mathrm{qv}\left(4 \mathrm{H}, \mathrm{CH}_{2}-\mathrm{Am}\right)$, $1.58 \mathrm{sc}\left(4 \mathrm{H}, \mathrm{CH}_{2}-\mathrm{Am}\right), 1.00 \mathrm{t}\left(6 \mathrm{H}, \mathrm{CH}_{3}-\mathrm{Am}\right),-3.20 \mathrm{bs}(2 \mathrm{H}, \mathrm{NH})$.

5-[4'-(10'"-Bromdecylcarbonyloxy) phenyl]-10,15,20triphenylporphine, 4 (para, $\mathrm{R}=\left(\mathrm{CH}_{2}\right)_{10} \mathrm{Br}$ ). A mixture of 5-(4'hydroxyphenyl)-10,15,20-triphenylporphine 1 (para) $(50 \mathrm{mg}$, $0.080 \mathrm{mmol}), 11$-bromoundecanoic acid $15\left(\mathrm{R}=\left(\mathrm{CH}_{2}\right)_{10} \mathrm{Br}\right)(23$ $\mathrm{mg}, 0.087 \mathrm{mmol})$, DMAP (6 mg, $0.048 \mathrm{mmol})$ and EDAC (23 $\mathrm{mg}, 0.119 \mathrm{mmol}$ ) in $15 \mathrm{ml}$ of dried dichloromethane was stirred firstly at cooling in the ice bath for 1.5 hours, and then at room temperature till the reaction completion ( $c a .3$ hours, TLC). The solution obtained was chromatographed on silica by $\mathrm{CH}_{2} \mathrm{Cl}_{2}$. Eluate was evaporated and the porphyrin was precipitated by methanol. The residue was filtered off, washed with methanol and dried at room temperature. Yield $50 \mathrm{mg}(71.6 \%) . R_{\mathrm{f}}=0.64$ (benzene). UVvis $\lambda_{\max } \mathrm{nm}(\lg \varepsilon): 648$ (3.92), 591 (3.89), 550 (4.00), 515 (4.31), 418 (5.69). IR $v \mathrm{~cm}^{-1}: 1757$ (-O-CO-). ${ }^{1} \mathrm{H}$ NMR $\delta$ ppm: $8.89 \mathrm{~m}(8 \mathrm{H}$, $\beta-\mathrm{H}), 8.25 \mathrm{~d}\left(8 \mathrm{H}, o-\mathrm{H}-\mathrm{Ph}+2^{\prime}, 6^{\prime}-\mathrm{H}-\mathrm{Ar}\right), 7.90 \mathrm{~m}(9 \mathrm{H}, m, p-\mathrm{H}-\mathrm{Ph})$, $7.53 \mathrm{~d}\left(2 \mathrm{H}, 3^{\prime}, 5^{\prime}-\mathrm{H}\right), 3.46 \mathrm{t}\left(2 \mathrm{H}, \mathrm{CH}_{2} \mathrm{CO}\right), 2.78 \mathrm{t}\left(2 \mathrm{H}, \mathrm{CH}_{2} \mathrm{Br}\right), 1.93$ 
$\mathrm{m}\left(4 \mathrm{H}, \mathrm{CH}_{2}\right), 1.48 \mathrm{~m}+1.40 \mathrm{~m}\left(12 \mathrm{H}, \mathrm{CH}_{2}\right),-2.75 \mathrm{bs}(2 \mathrm{H}, \mathrm{NH})$. 5-[4'-(4"'-n-Dodecyloxyphenylcarbonyloxy) phenyl]10,15,20-triphenylporphine, 4 (para, $\mathrm{R}=4-\mathrm{C}_{6} \mathrm{H}_{4} \mathrm{O}\left(\mathrm{CH}_{2}\right)_{11} \mathrm{CH}_{3}$ ). A mixture of 5-(4'-hydroxyphenyl)-10,15,20-triphenylporphine 1 (para) (50 mg, $0.080 \mathrm{mmol}), 4-n$-dodecyloxybenzoic acid $15\left(\mathrm{R}=4-\mathrm{C}_{6} \mathrm{H}_{4} \mathrm{O}\left(\mathrm{CH}_{2}\right)_{11} \mathrm{CH}_{3}\right)(49 \mathrm{mg}, 0.160 \mathrm{mmol})$, DMAP (6 $\mathrm{mg}, 0.049 \mathrm{mmol}$ ) and EDAC (31 mg, $0.162 \mathrm{mmol})$ in $15 \mathrm{ml}$ of dried dichloromethane was stirred firstly in an ice bath for 1.5 hours, and then at room temperature till the reaction completion ( $c a .3$ hours, TLC). The product was isolated on the next day analogously the previous procedure. Yield $72 \mathrm{mg}(98 \%) . R_{\mathrm{f}}=$ 0.65 (benzene). UV-vis $\lambda_{\text {max }}$ nm (lge): 648 (3.75), 590 (3.74), 550 (3.87), 515 (4.16), 418 (5.56). IR $v \mathrm{~cm}^{-1}: 1734$ (-O-CO-). ${ }^{1} \mathrm{H}$ NMR $\delta$ ppm: $8.90 \mathrm{~m}(8 \mathrm{H}, \beta-\mathrm{H}), 8.35 \mathrm{~d}\left(2 \mathrm{H}, 2^{\prime}, 6\right.$ '-H-Ar $), 8.26 \mathrm{~m}$ (6H, o-H-Ph), $8.15 \mathrm{~d}(2 \mathrm{H}, 2 ", 6 "-\mathrm{H}-\mathrm{Ar}), 7.80 \mathrm{~m}(9 \mathrm{H}, m, p-\mathrm{H}-\mathrm{Ph})$, $7.08 \mathrm{~d}(2 \mathrm{H}, 3$ ', 5'-H-Ar), $6.95 \mathrm{~d}(2 \mathrm{H}, 3$ ", 5"-H-Ar), $4.04 \mathrm{t}(2 \mathrm{H}$, $\left.\mathrm{OCH}_{2}\right), 1.82 \mathrm{~m}\left(2 \mathrm{H}, \mathrm{CH}_{2}\right), 1.52 \mathrm{~m}\left(2 \mathrm{H}, \mathrm{CH}_{2}\right), 1.33 \mathrm{~m}\left(16 \mathrm{H}, \mathrm{CH}_{2}\right)$, $0.93 \mathrm{t}\left(3 \mathrm{H}, \mathrm{CH}_{3}\right),-2.72$ bs $(2 \mathrm{H}, \mathrm{NH})$.

5-[4'-(N-tert-Butyloxycarbonylglycyloxy) phenyl]-10,15,20triphenylporphine, 4 (para, $\mathrm{R}=\mathrm{CH}_{2} \mathrm{NH}-\mathrm{BOC}$ ). A mixture of 5-(4'oxyphenyl)-10,15,20-triphenylporphine 1 (para) (50 mg, 0.080 mmol), $N$-tert-butyloxycarbonylglycine $15\left(\mathrm{R}=\mathrm{CH}_{2} \mathrm{NH}-\mathrm{BOC}\right)(27$ $\mathrm{mg}, 0.158 \mathrm{mmol}$ ), DMAP (6 mg, $0.049 \mathrm{mmol})$ and EDAC (30 mg, $0.156 \mathrm{mmol}$ ) in $15 \mathrm{ml}$ of dry dichloromethane was stirred firstly in an ice bath for 1.5 hours, and then at room temperature till the reaction completion ( $c a .3$ hours, TLC). The product was isolated on the next day analogously to the above procedure. Yield $42.5 \mathrm{mg}$ $(68 \%) . R_{\mathrm{f}}=0.81$ (benzene-methanol, 10:1). UV-vis $\lambda_{\text {max }} \mathrm{nm}(\lg \varepsilon)$ : 648 (3.81), 590 (3.82), 550 (3.95), 515 (4.24), 418 (5.58). IR $v \mathrm{~cm}^{-1}$ : 1771 (-O-CO-). ${ }^{1} \mathrm{H}$ NMR $\delta$ ppm: $8.87 \mathrm{~m}(8 \mathrm{H}, \beta-\mathrm{H}), 8.25 \mathrm{~m}(6 \mathrm{H}$, $o-\mathrm{H}-\mathrm{Ph}), 8.11 \mathrm{~d}\left(2 \mathrm{H}, 2^{\prime}, 6\right.$ '-H-Ar), $7.78 \mathrm{~m}(9 \mathrm{H}, m, p-\mathrm{H}-\mathrm{Ph}), 7.54 \mathrm{~d}$ $\left(2 \mathrm{H}, 3\right.$ ', 5'-H-Ar), 5.24 bs $(1 \mathrm{H}, \mathrm{NH}), 4.39 \mathrm{~d}\left(2 \mathrm{H}, \mathrm{CH}_{2}\right), 1.57 \mathrm{~s}(9 \mathrm{H}$, $\left.{ }^{t} \mathrm{Bu}\right),-2.76$ bs $(2 \mathrm{H}, \mathrm{NH})$.

5-[3'-(10"-Bromdecylcarbonyloxy) phenyl]-10,15,20-triphenylporphine, 4 (meta, $\mathrm{R}=\left(\mathrm{CH}_{2}\right)_{10} \mathrm{Br}$ ). A mixture of 5-(3-oxyphenyl)-10,15,20-triphenylporphine 1 (meta) $(50 \mathrm{mg}, 0.079 \mathrm{mmol})$, 11-bromoundecanoic acid $15\left(\mathrm{R}=\left(\mathrm{CH}_{2}\right)_{10} \mathrm{Br}\right)(23 \mathrm{mg}, 0.087 \mathrm{mmol})$, DMAP (6 mg, $0.048 \mathrm{mmol})$ and EDAC (23 mg, $0.120 \mathrm{mmol})$ in 15 $\mathrm{ml}$ of dried dichloromethane was stirred firstly in an ice bath for 1.5 hours, and then at room temperature till the reaction completion ( $c a$. 3 hours, TLC). The product was isolated on the next day analogously to the above procedure. Yield $34.3 \mathrm{mg}(49.4 \%) . R_{\mathrm{f}}=0.41$ (benzene). UV-vis $\lambda_{\text {max }}$ nm (lge): 648 (3.86), 590 (3.86), 549 (3.97), 515 (4.28), 418 (5.66). IR $v \mathrm{~cm}^{-1}: 1632$ (-O-CO-). ${ }^{1} \mathrm{H}$ NMR $\left(\mathrm{CDCl}_{3}\right) \delta \mathrm{ppm}: 8.91$ $\mathrm{d}(2 \mathrm{H}, \beta-\mathrm{H}), 8.87 \mathrm{~s}(6 \mathrm{H}, \beta-\mathrm{H}), 8.24 \mathrm{~d}(1 \mathrm{H}, 6-\mathrm{H}-\mathrm{Ar}), 7.83 \mathrm{~d}(6 \mathrm{H}, o-\mathrm{H}-$ $\mathrm{Ph}), 7.79 \mathrm{~m}(10 \mathrm{H}, m, p-\mathrm{H}-\mathrm{Ph}, 2-\mathrm{H}-\mathrm{Ar}), 7.65 \mathrm{t}(1 \mathrm{H}, 5-\mathrm{H}-\mathrm{Ar}), 7.34 \mathrm{~d}$ $(1 \mathrm{H}, 4-\mathrm{H}-\mathrm{Ar}), 3.36 \mathrm{t}\left(2 \mathrm{H}, \mathrm{CH}_{2} \mathrm{Br}\right), 2.29 \mathrm{t}\left(2 \mathrm{H}, \mathrm{COCH}_{2}\right), 1.96 \mathrm{~m}(2 \mathrm{H}$, $\left.\mathrm{CH}_{2}\right), 1.91 \mathrm{~m}\left(2 \mathrm{H}, \mathrm{CH}_{2}\right), 1.80 \mathrm{qv}\left(2 \mathrm{H}, \mathrm{CH}_{2}\right), 1.60 \mathrm{qv}\left(2 \mathrm{H}, \mathrm{CH}_{2}\right), 1.36$ qv $\left(2 \mathrm{H}, \mathrm{CH}_{2}\right), 1.23 \mathrm{~s}\left(6 \mathrm{H}, \mathrm{CH}_{2}\right),-2.75 \mathrm{bs}(2 \mathrm{H}, \mathrm{NH})$.

5-[2'-(10”-Bromdecylcarbonyloxy) phenyl]-10,15,20triphenylporphine, 4 (ortho, $\mathrm{R}=\left(\mathrm{CH}_{2}\right)_{10} \mathrm{Br}$ ). A mixture of 5-(2hydroxyphenyl)-10,15,20-triphenylporphine $\mathbf{1}$ (ortho) (50 mg, $0.080 \mathrm{mmol}), 11$-bromoundecanoic acid $\mathbf{1 5}\left(\mathrm{R}=\left(\mathrm{CH}_{2}\right)_{10} \mathrm{Br}\right)(23 \mathrm{mg}$, $0.086 \mathrm{mmol}$ ), DMAP (6 mg, $0.048 \mathrm{mmol}$ ) and EDAC (23 mg, 0.120 $\mathrm{mmol}$ ) in $15 \mathrm{ml}$ of dried dichloromethane was stirred firstly in an ice bath for 1.5 hours, and then at room temperature till the reaction completion ( $c a .3$ hours, TLC). The product was isolated on the next day analogously the methodology mentioned above. Yield $32.8 \mathrm{mg}(46.5 \%) . R_{\mathrm{f}}=0.84$ (benzene). UV-vis $\lambda_{\max } \mathrm{nm}(\lg \varepsilon): 650$ (4.02), 597 (3.68), 555 (3.71), 517 (3.98), 418 (5.13). IR $v \mathrm{~cm}^{-1}$ : 1625 (-O-CO-). ${ }^{1} \mathrm{H}$ NMR $\delta$ ppm: $8.87 \mathrm{~m}(6 \mathrm{H}, \beta-\mathrm{H}), 8.83 \mathrm{~m}(2 \mathrm{H}$, $\beta-\mathrm{H}), 8.19 \mathrm{~m}(6 \mathrm{H}, o-\mathrm{H}-\mathrm{Ph}), 7.76 \mathrm{~d}\left(1 \mathrm{H}, 6^{\prime}-\mathrm{H}-\mathrm{Ar}\right), 7.71 \mathrm{t}\left(1 \mathrm{H}, 4^{\prime}-\right.$ $\mathrm{H}-\mathrm{Ar}), 7.69 \mathrm{~m}(9 \mathrm{H}, m, p-\mathrm{H}-\mathrm{Ph}), 7.37 \mathrm{~d}\left(1 \mathrm{H}, 3^{\prime}-\mathrm{H}-\mathrm{Ar}\right), 7.32 \mathrm{t}(1 \mathrm{H}$, 5'-H-Ar), 3.25 t (2H, CH $\mathrm{Br}), 1.35 \mathrm{t}\left(2 \mathrm{H}, \mathrm{COCH}_{2}\right), 1.07 \mathrm{~m}\left(\mathrm{CH}_{2}\right)$, $0.57 \mathrm{~m}\left(\mathrm{CH}_{2}\right), 0.49 \mathrm{~m}\left(\mathrm{CH}_{2}\right), 0.40 \mathrm{~m}\left(\mathrm{CH}_{2}\right), 0.27 \mathrm{~m}\left(\mathrm{CH}_{2}\right), 0.13 \mathrm{~m}$ $\left(\mathrm{CH}_{2}\right),-0.01 \mathrm{~m}\left(\mathrm{CH}_{2}\right),-0.20 \mathrm{~m}\left(\mathrm{CH}_{2}\right),-2.76$ bs $(2 \mathrm{H}, \mathrm{NH})$.

5-[4'-(10"-Bromdecylcarbonyloxy)phenyl]-2,3, 7,8, 12,18hexamethyl-13,17-di-n-amylporphine, 6 (para, $\mathrm{R}=\left(\mathrm{CH}_{2}\right)_{10} \mathrm{Br}$ ).
A mixture of 5-(4'-oxyphenyl)-2,3,7,8,12,18-hexamethyl-13,17diamylporphine 3 (para) (49 mg, $0.078 \mathrm{mmol}$ ), 11-bromoundecanoic acid $(23 \mathrm{mg}, 0.086 \mathrm{mmol})$, DMAP ( $6 \mathrm{mg}, 0.048 \mathrm{mmol})$ and EDAC (23 mg, $0.120 \mathrm{mmol}$ ) in $15 \mathrm{ml}$ of dry dichloromethane was stirred firstly in an ice bath for 1.5 hours, and then at room temperature till the reaction completion ( $c a .3$ hours, TLC). The product was isolated by chromatography over silica (eluent $-\mathrm{CH}_{2} \mathrm{Cl}_{2}$ ). The solvent was distilled off on a rotary evaporator; the residue was precipitated by methanol. Yield $51 \mathrm{mg}(74.7 \%) . R_{\mathrm{f}}=0.79$ (benzene). UV-vis $\lambda$ $\mathrm{nm}$ (lge): 624 (3.62), 571 (3.92), 537 (3.95), 503 (4.24), 403 (5.34). IR $v \mathrm{~cm}^{-1}: 1736$ (-O-CO-). ${ }^{1} \mathrm{H}$ NMR $\delta$ ppm: $10.17 \mathrm{~s}(2 \mathrm{H}, 10,20-\mathrm{H})$, $9.97 \mathrm{~s}(1 \mathrm{H}, 15-\mathrm{H}), 8.06 \mathrm{~d}\left(2 \mathrm{H}, 2^{\prime}, 6^{\prime}-\mathrm{H}-\mathrm{Ar}\right), 7.48 \mathrm{~d}\left(2 \mathrm{H}, 3^{\prime}, 5^{\prime}-\mathrm{H}-\mathrm{Ar}\right)$, $4.05 \mathrm{t}\left(4 \mathrm{H}, \mathrm{CH}_{2}-\mathrm{Am}\right), 3.65 \mathrm{~s}\left(6 \mathrm{H}, 12,18-\mathrm{CH}_{3}\right), 3.55 \mathrm{~s}\left(6 \mathrm{H}, 2,8-\mathrm{CH}_{3}\right)$, $3.46 \mathrm{t}\left(2 \mathrm{H}, \mathrm{COCH}_{2}\right), 2.78 \mathrm{t}\left(2 \mathrm{H}, \mathrm{CH}_{2} \mathrm{Br}\right), 2.53 \mathrm{~s}\left(6 \mathrm{H}, 3,7-\mathrm{CH}_{3}\right), 2.32$ qv $\left(4 \mathrm{H}, \mathrm{CH}_{2}-\mathrm{Am}\right), 1.93 \mathrm{~m}\left(6 \mathrm{H}, \mathrm{CH}_{2}, \mathrm{CH}_{2}-\mathrm{Am}\right), 1.75 \mathrm{qv}\left(4 \mathrm{H}, \mathrm{CH}_{2}-\right.$ $\mathrm{Am}), 1.58 \mathrm{sc}\left(4 \mathrm{H}, \mathrm{CH}_{2}-\mathrm{Am}\right), 1.48 \mathrm{~m}\left(4 \mathrm{H}, \mathrm{CH}_{2}\right), 1.41 \mathrm{~m}\left(10 \mathrm{H}, \mathrm{CH}_{2}\right)$, $0.99 \mathrm{t}\left(6 \mathrm{H}, \mathrm{CH}_{3}-\mathrm{Am}\right),-3.17 \mathrm{bs},-3.31 \mathrm{bs}(2 \times 1 \mathrm{H}, \mathrm{NH})$.

5-[3'-(10'-Bromdecylcarbonyloxy)phenyl]-2,3, 7, 8, 12,18hexamethyl-13,17-di-n-amylporphine, 6 (meta, $\mathrm{R}=\left(\mathrm{CH}_{2}\right)_{10} \mathrm{Br}$ ). A mixture of 5-(3'-hydroxyphenyl)-2,3,7,8,12,18-hexamethyl-13,17di-amylporphine 3 (meta) $(50 \mathrm{mg}, 0.080 \mathrm{mmol}), 11$-bromoundecanoic acid $(23 \mathrm{mg}, 0.086 \mathrm{mmol})$, DMAP $(6 \mathrm{mg}, 0.048 \mathrm{mmol})$ and EDAC $(23 \mathrm{mg}, 0.120 \mathrm{mmol})$ in $15 \mathrm{ml}$ of dried dichloromethane was stirred firstly in an ice bath for 1.5 hours, and then at room temperature till the reaction completion ( $c a .3$ hours, TLC). The product was isolated by chromatography on silica (eluent $-\mathrm{CH}_{2} \mathrm{Cl}_{2}$ ). The solvent was distilled off on a rotary evaporator; the residue was precipitated by methanol. Yield $62.8 \mathrm{mg}(90 \%) . R_{\mathrm{f}}=0.86$ (benzene). UV-vis $\lambda_{\mathrm{m}}$ nm (lge): 623 (3.67), 571 (3.94), 537 (3.98), 503 (4.24), 403 (5.32). IR $v \mathrm{~cm}^{-1}$ : 1758 (-O-CO-). ${ }^{1} \mathrm{H}$ NMR $\delta$ ppm: $10.18 \mathrm{~s}(2 \mathrm{H}, 10,20-\mathrm{H})$, $9.97 \mathrm{~s}(1 \mathrm{H}, 15-\mathrm{H}), 7.94 \mathrm{~d}\left(1 \mathrm{H}, 6{ }^{\prime}-\mathrm{H}-\mathrm{Ar}\right), 7.82 \mathrm{~s}\left(1 \mathrm{H}, 2^{\prime}-\mathrm{H}-\mathrm{Ar}\right)$, $7.74 \mathrm{t}\left(1 \mathrm{H}, 5\right.$ '-H-Ar), $7.53 \mathrm{~d}\left(1 \mathrm{H}, 4^{\prime}-\mathrm{H}-\mathrm{Ar}\right), 4.05$ t (4H, $\left.\mathrm{CH}_{2}-\mathrm{Am}\right)$, $3.65 \mathrm{~s}\left(6 \mathrm{H}, 12,18-\mathrm{CH}_{3}\right), 3.56 \mathrm{~s}\left(6 \mathrm{H}, 2,8-\mathrm{CH}_{3}\right), 3.34 \mathrm{t}\left(2 \mathrm{H}, \mathrm{COCH}_{2}\right)$, $2.65 \mathrm{t}\left(2 \mathrm{H}, \mathrm{CH}_{2} \mathrm{Br}\right), 2.58 \mathrm{~s}\left(6 \mathrm{H}, 3,7-\mathrm{CH}_{3}\right), 2.33 \mathrm{qv}\left(4 \mathrm{H}, \mathrm{CH}_{2}-\mathrm{Am}\right)$, $1.76 \mathrm{~m}\left(6 \mathrm{H}, \mathrm{CH}_{2}, \mathrm{CH}_{2}-\mathrm{Am}\right), 1.57 \mathrm{sc}\left(4 \mathrm{H}, \mathrm{CH}_{2}-\mathrm{Am}\right), 1.44 \mathrm{~m}(2 \mathrm{H}$, $\left.\mathrm{CH}_{2}\right), 1.34 \mathrm{~m}\left(2 \mathrm{H}, \mathrm{CH}_{2}\right), 1.27 \mathrm{~m}\left(10 \mathrm{H}, \mathrm{CH}_{2}\right), 1.00 \mathrm{t}\left(6 \mathrm{H}, \mathrm{CH}_{3}-\mathrm{Am}\right)$, $-3.19 \mathrm{bs},-3.32$ bs $(2 \times 1 \mathrm{H}, \mathrm{NH})$.

5-[2'-(10'-Bromdecylcarbonyloxy)phenyl]-2,3, 7, 8, 12,18hexamethyl-13,17-di-n-amylporphine, 6 (ortho, $\mathrm{R}=\left(\mathrm{CH}_{2}\right){ }_{10} \mathrm{Br}$ ). A mixture of 5-(2'-hydroxyphenyl)-2,3,7,8,12,18-hexamethyl-13,17diamylporphine 3 (ortho) $(50 \mathrm{mg}, 0.080 \mathrm{mmol}$ ), 11-bromoundecanoic acid $(23 \mathrm{mg}, 0.086 \mathrm{mmol})$, DMAP ( $6 \mathrm{mg}, 0.048 \mathrm{mmol})$ and EDAC (23 mg, $0.120 \mathrm{mmol}$ ) in $15 \mathrm{ml}$ of dry dichloromethane was stirred firstly in an ice bath for 1.5 hours, and then at room temperature till the reaction completion ( $\mathrm{ca} .3$ hours, TLC). The product was isolated by chromatography on silica (eluent $-\mathrm{CH}_{2} \mathrm{Cl}_{2}$ ). The solvent was distilled off on a rotary evaporator; the residue was precipitated by methanol. Yield $62.8 \mathrm{mg}(90 \%) . R_{\mathrm{f}}=0.88$ (benzene). UV-vis $\lambda_{\text {max }}$ nm (lge): 624 (3.64), 571 (3.89), 536 (3.95), 502 (4.22), 403 (5.31). IR $v \mathrm{~cm}^{-1}: 1756$ (-O-CO-). ${ }^{1} \mathrm{H}$ NMR $\delta$ ppm: $10.17 \mathrm{~s}(2 \mathrm{H}, 10,20-\mathrm{H})$, $9.98 \mathrm{~s}(1 \mathrm{H}, 15 \mathrm{H}), 8.10 \mathrm{~d}(1 \mathrm{H}, 6-\mathrm{H}-\mathrm{Ar}), 7.86 \mathrm{t}(1 \mathrm{H}, 4-\mathrm{H}-\mathrm{Ar}), 7.65$ $\mathrm{m}(2 \mathrm{H}, 2,5-\mathrm{H}-\mathrm{Ar}), 4.06 \mathrm{t}\left(4 \mathrm{H}, \mathrm{CH}_{2}-\mathrm{Am}\right), 3.66 \mathrm{~s}\left(6 \mathrm{H}, 12,18-\mathrm{CH}_{3}\right)$, $3.56 \mathrm{~s}\left(6 \mathrm{H}, 2,8-\mathrm{CH}_{3}\right), 3.25 \mathrm{t}\left(2 \mathrm{H}, \mathrm{CH}_{2} \mathrm{Br}\right), 2.58 \mathrm{~s}\left(6 \mathrm{H}, 3,7-\mathrm{CH}_{3}\right)$, 2.33 qv (4H, $\left.\mathrm{CH}_{2}-\mathrm{Am}\right), 1.76 \mathrm{qv}\left(4 \mathrm{H}, \mathrm{CH}_{2}-\mathrm{Am}\right), 1.57 \mathrm{~m}\left(8 \mathrm{H}, \mathrm{CH}_{2}-\right.$ $\left.\mathrm{Am}, \mathrm{CH}_{2}\right), 1.28 \mathrm{t}^{2}\left(2 \mathrm{H}, \mathrm{CH}_{2} \mathrm{CO}\right), 1.00 \mathrm{t}\left(6 \mathrm{H}, \mathrm{CH}_{3}-\mathrm{Am}\right), 0.95 \mathrm{qv}(2 \mathrm{H}$, $\left.\mathrm{CH}_{2}\right), 0.50$ qv $\left(2 \mathrm{H}, \mathrm{CH}_{2}\right), 0.1 \mathrm{qv}\left(2 \mathrm{H}, \mathrm{CH}_{2}\right), 0.01 \mathrm{qv}\left(2 \mathrm{H}, \mathrm{CH}_{2}\right)$, $-0.19 \mathrm{qv}\left(2 \mathrm{H}, \mathrm{CH}_{2}\right),-0.29 \mathrm{qv}\left(2 \mathrm{H}, \mathrm{CH}_{2}\right),-0.56 \mathrm{qv}\left(2 \mathrm{H}, \mathrm{CH}_{2}\right),-3.14$ bs $(1 \mathrm{H}, \mathrm{NH}),-3.29$ bs $(1 \mathrm{H}, \mathrm{NH})$.

5-[4'-(N-tert-Butyloxycarbonyl-L-phenylalanyloxy)phenyl]2,3,7,8,12,18-hexamethyl-13,17-di-n-amylporhine, 6 (para, $R=$ $\left.\mathrm{CH}(\mathrm{NH}-\mathrm{BOC}) \mathrm{CH}_{2} \mathrm{C}_{6} \mathrm{H}_{5}\right)$. A mixture of 5-(4'-hydroxyphenyl)2,3,7,8,12,18-hexamethyl-13,17-di-n-amylporhine 3 (para) $(50$ $\mathrm{mg}, 0.080 \mathrm{mmol}), N$-tert-butyloxycarbonyl- $L$-phenylalanine $15(\mathrm{R}$ $\left.=\mathrm{CH}(\mathrm{NH}-\mathrm{BOC}) \mathrm{CH}_{2} \mathrm{C}_{6} \mathrm{H}_{5}\right)(42 \mathrm{mg}, 0.158 \mathrm{mmol})$, DMAP $(6 \mathrm{mg}$, $0.049 \mathrm{mmol})$ and EDAC (30 mg, $0.156 \mathrm{mmol})$ in $15 \mathrm{ml}$ of dry dichloromethane was stirred firstly in an ice bath for 1.5 hours, and then at room temperature. The reaction proceeded comparatively slowly, and the whole reaction time was 3 days. The product was 
isolated analogously the methodology, mentioned above. Yield 38 $\mathrm{mg}(52.4 \%) . R_{\mathrm{f}}=0.83$ (benzene-methanol, 10:1). UV-vis $\lambda_{\max } \mathrm{nm}$ (lge): 623 (3.61), 579 (3.82), 538 (3.94), 503 (4.21), 404 (5.30). IR $v \mathrm{~cm}^{-1}: 1631$ (-O-CO-). ${ }^{1} \mathrm{H}$ NMR $\left(\mathrm{CDCl}_{3}\right) \delta \mathrm{ppm}: 10.19 \mathrm{~s}(2 \mathrm{H}$, $10,20-\mathrm{H}), 9.99 \mathrm{~s}(1 \mathrm{H}, 15-\mathrm{H}), 8.06 \mathrm{~d}(2 \mathrm{H}, o-\mathrm{H}-\mathrm{Ar}), 7.44 \mathrm{~m}(7 \mathrm{H}$, $m$-H-Ar, Ph-Al), $5.25 \mathrm{~d}(1 \mathrm{H}, \mathrm{NH}), 5.04 \mathrm{q}(1 \mathrm{H}, \mathrm{CH}), 3.95 \mathrm{t}(4 \mathrm{H}$, $\left.\mathrm{CH}_{2}-\mathrm{Am}\right), 3.66 \mathrm{~s}\left(6 \mathrm{H}, 12,18-\mathrm{CH}_{3}\right), 3.56 \mathrm{~s}\left(6 \mathrm{H}, 2,8-\mathrm{CH}_{3}\right), 3.44 \mathrm{~d}$ $\left(2 \mathrm{H}, \mathrm{CH}_{2}\right), 2.51 \mathrm{~s}\left(6 \mathrm{H}, 2,7-\mathrm{CH}_{3}\right), 2.28 \mathrm{qv}\left(4 \mathrm{H}, \mathrm{CH}_{2}-\mathrm{Am}\right), 1.72 \mathrm{qv}$ $\left(4 \mathrm{H}, \mathrm{CH}_{2}-\mathrm{Am}\right), 1.53 \mathrm{~m}\left(4 \mathrm{H}, \mathrm{CH}_{2}-\mathrm{Am}\right), 1.55 \mathrm{~s}\left(9 \mathrm{H},{ }^{t} \mathrm{Bu}\right), 0.98 \mathrm{t}(6 \mathrm{H}$, $\left.\mathrm{CH}_{3}-\mathrm{Am}\right),-3.26$ bs $(2 \mathrm{H}, \mathrm{NH})$.

5,10,15,20-Tetrakis [4'-(10"'-bromdecylcarbonyloxy)phenyl] porphine, 5 (para, $\mathrm{R}=\left(\mathrm{CH}_{2}\right.$ ) $\mathrm{Br}$ ). a) A mixture of 5,10,15,20tetrakis(4'-hydroxyphenyl)porphine 2 (para) $(50 \mathrm{mg}, 0.073 \mathrm{mmol}$ ), 11-bromoundecanoic acid ( $86 \mathrm{mg}, 0.325 \mathrm{mmol})$, DMAP (21 mg, $0.175 \mathrm{mmol})$ and $\operatorname{EDAC}(85 \mathrm{mg}, 0.442 \mathrm{mmol})$ in $15 \mathrm{ml}$ of dry dichloromethane was stirred at $0{ }^{\circ} \mathrm{C}$ for 1.5 hours; then the reaction mixture was refluxed during 2 days because of a poor solubility of the initial porphyrin. The product was isolated analogously the methodology, mentioned above. Yield $20 \mathrm{mg}(16.4 \%)$.

b) A mixture of 5,10,15,20-tetrakis(4'-hydroxyphenyl)porphine 2 (para) (50 mg, $0.073 \mathrm{mmol})$, 11-bromoundecanoic acid ( $86 \mathrm{mg}, 0.325 \mathrm{mmol})$, DMAP (21 mg, $0.175 \mathrm{mmol}$ ) and EDAC (85 $\mathrm{mg}, 0.442 \mathrm{mmol}$ ) in $15 \mathrm{ml}$ of dried tetrahydrofuran was stirred firstly in an ice bath for 1.5 hours and then 3 days at room temperature. THF was distilled off, the residue was dissolved in dichloromethane and chromatographed on silica. The first porphyrin fraction was collected, the solvent was distilled off, and the porphyrin was precipitated by methanol. Yield $24.4 \mathrm{mg}(20.4 \%)$.

$R_{f}=0.77$ (benzene). UV-vis $\lambda_{\max } \mathrm{nm}(\lg \varepsilon): 646$ (3.75), 590 (3.91), 551 (4.06), 515 (4.41), 420 (5.72). ${ }^{1} \mathrm{H}$ NMR $\delta$ ppm: $8.98 \mathrm{~s}$ $(8 \mathrm{H}, \beta-\mathrm{H}), 8.26 \mathrm{~d}\left(8 \mathrm{H}, 2^{\prime}, 6^{\prime}-\mathrm{H}-\mathrm{Ar}\right), 7.55 \mathrm{~d}\left(8 \mathrm{H}, 3^{\prime}, 5^{\prime}-\mathrm{H}\right), 3.46 \mathrm{t}(8 \mathrm{H}$, $\left.\mathrm{CH}_{2} \mathrm{CO}\right), 2.78 \mathrm{t}\left(8 \mathrm{H}, \mathrm{CH}_{2} \mathrm{Br}\right), 1.97 \mathrm{~m}\left(16 \mathrm{H}, \mathrm{CH}_{2}\right), 1.48 \mathrm{~m}, 1.40 \mathrm{~m}$ $\left(48 \mathrm{H}, \mathrm{CH}_{2}\right)-2.75$ bs $(2 \mathrm{H}, \mathrm{NH})$.

5,10,15,20-Tetrakis [3'-(10"'-bromdecylcarbonyloxy)phenyl] porphine, 5 (meta, $\mathrm{R}=\left(\mathrm{CH}_{2}\right)_{10} \mathrm{Br}$ ). A mixture of 5,10,15,20tetrakis(4'-hydroxyphenyl)porphine 2 ( $m e t a)$ ( $50 \mathrm{mg}, 0.073 \mathrm{mmol}$ ), 11-bromoundecanoic acid (86 mg, $0.325 \mathrm{mmol})$, DMAP (22 mg, $0.179 \mathrm{mmol}$ ) and EDAC ( $85 \mathrm{mg}, 0.442 \mathrm{mmol})$ in $15 \mathrm{ml}$ of dried dichloromethane was stirred at $0{ }^{\circ} \mathrm{C}$ for 1.5 hours; then the reaction mixture was refluxed during 1 day because of a poor solubility of the initial porphyrin. The product was isolated analogously the methodology, mentioned above. Yield $18.2 \mathrm{mg}(14.5 \%) . R_{\mathrm{f}}=0.85$ (benzene). UV-vis $\lambda_{\text {max }} \mathrm{nm}$ (lge): 645 (3.66), 589 (3.79), 549 (3.85), 514 (4.19), 418 (5.54). ${ }^{1} \mathrm{H}$ NMR $\delta$ ppm: $8.96 \mathrm{~s}(8 \mathrm{H}, \beta-\mathrm{H}), 8.09 \mathrm{~d}$ (4H, 6'-H-Ar), 7.99 s (4H, 2'-H-Ar), 7.79 t (4H, 5'-H-Ar), $7.57 \mathrm{~d}$ $\left(4 \mathrm{H}, 4^{\prime}-\mathrm{H}-\mathrm{Ar}\right), 3.35 \mathrm{t}\left(8 \mathrm{H}, \mathrm{CH}_{2} \mathrm{Br}\right), 2.68 \mathrm{t}\left(8 \mathrm{H}, \mathrm{COCH}_{2}\right), 1.82 \mathrm{qv}$ $\left(16 \mathrm{H}, \mathrm{CH}_{2}\right), 1.45 \mathrm{~m}\left(8 \mathrm{H}, \mathrm{CH}_{2}\right), 1.37 \mathrm{~m}\left(8 \mathrm{H}, \mathrm{CH}_{2}\right), 1.29 \mathrm{~m}(32 \mathrm{H}$, $\left.\mathrm{CH}_{2}\right),-2.84$ bs $(2 \mathrm{H}, \mathrm{NH})$.

\section{References}

1. Golubchikov O.A. In: Porfiriny: Spektroscopiya, Elektrokhimiya, Primenenie [Porphyrins: Spectroscopy, Electrochemistry, Application] (Enikolopyan N.S., Ed.) Moscow: Nauka. 1987, 384 p. (in Russ.).

2. Reshetnikov A.V., Shvetz V.I., Ponomarev G.V. In: Uspekhi Khimii Porfirinov [The Advances in Porpyrin Chemistry]. Vol. 2 (Golubchikov O.A., Ed.) St. Petersburg: NII Khimii SPbGU 1999, 70-114 (in Russ.).

3. Evstigneeva R.P. Uspekhi Khimii Porfirinov [The Advances in Porpyrin Chemistry]. Vol. 2 (Golubchikov O.A., Ed.) StPetersburg, NII Khimii SPbGU 1999, 115-127 (in Russ.).

4. Kurek S.S. In: Uspekhi Khimii Porfirinov [The Advances in Porpyrin Chemistry]. Vol. 2 (Golubchikov O.A., Ed.) St.
Petersburg: NII Khimii SPbGU. 1999, 223-241 (in Russ.).

5. Evstigneeva R.P. In: Uspekhi Khimii Porfirinov [The Advances in Porpyrin Chemistry]. Vol. 3 (Golubchikov O.A., Ed.) St. Petersburg: NII Khimii SPbGU. 2001, 150-159 (in Russ.).

6. Solov'ova A.B., Rumyntzeva T.N. In: Uspekhi Khimii Porfirinov [The Advances in Porpyrin Chemistry]. Vol. 3 (Golubchikov O.A., Ed.) St-Petersburg: NII Khimii SPbGU. 2001, 245-259 (in Russ.).

7. Bykova V.V., Usol'tzeva N.V., Semeikin A.S., Anan'eva G.A., Karmanova T.V. Zhidkie Kristalli i ikh Prakticheskoe Ispol'zovanie [Liquid Crystals and Their Practical Application] 2007, Issue 4 (22), 67-74. (in Russ.).

8. Bykova V.V., Usol'tzeva N.V., Semeikin A.S., Anan'eva G.A., Karmanova T.V., Lubimova T.V. Zhidkie Kristalli $i$ ikh Prakticheskoe Ispol'zovanie [Liquid Crystals and Their Practical Application] 2008, Issue 2 (24), 28-34. (in Russ.).

9. Syrbu S.A., Semeikin A.S., Berezin B.D., Koifman O.I. Khim. Geterotsikl. Soedin. 1989, 1373-1377 (in Russ.) [Chem. Heterocycl. Compd. 1989, 1149-1153 (engl. transl.)].

10. Syrbu S.A., Semeikin A.S., Koifman O.I., Berezin B.D. Khim. Geterotsikl. Soedin. 1987, 781-786 (in Russ.) [Chem. Heterocycl. Compd. 1987, 645-650 (engl. transl.)].

11. Kamachi M., Cheng X.S., Kida T., Kajiwara A., Shibasaka M., Nagata S. Macromolecules 1987, 20, 2665-2669.

12. Yu M., Liu G.F., Cui X.L. J. Porphyrins Phthalocyanines 2005, 9, 231-239.

13. Gerasimova O.A., Milaeva E.R., Shpakovsky D.B., Semeikin A.S., Syrbu S.A. Russ. Chem. Bull., Int. Ed. 2007, 56, 831-834 [Izv. Akad. Nauk, Ser. Khim. 2007, 56, 800-803, in Russ.].

14. Matile S., Hansen T., Stocker A., Woggon W.-D. Helv. Chim. Acta 1994, 77, 1087-1098.

15. Mehta G., Muthusamy S., Maiya B.G., Sirish M. J. Chem. Soc., Perkin Trans. I. 1996, 2421-2423.

16. Zimmerman S.C., Zharov I., Wendland M.S., Rakow N.A., Suslick K.S. J. Am. Chem. Soc. 2003, 125, 13504-13518.

17. Bhyrappa P., Vaijayanthimala G., Suslick K.S. J. Am. Chem. Soc. 1999, 121, 262-263.

18. Wang Q.M., Bruce D.W. Chem. Commun. 1996, 2505-2506.

19. Lindsey J.S., Scheriman I.C., Hsu H.C., Kearney P.C., Marguerettaz A.M. J. Org. Chem. 1987, 52, 827-836.

20. Lindsey J.S., Hsu H.C., Schreiman I.C. Tetrahedron Lett. 1986, 27, 4969-4970.

21. Lindsey J.S., MacCrum K.A., Tyhonas J.S., Chuang Y.-Y. J. Org. Chem. 1994, 59, 579-587.

22. Lindsey J.S., Wagner R.W. J. Org. Chem. 1989, 54, 828-836.

23. Li F., Yang K., Tyhonas J.S., MacCrum K.A., Lindsey J.S Tetrahedron 1997, 53, 12339-12360.

24. van der Made A.W., Hoppenbrouwer E.J.H., Nolte R.J.M., Drenth W. Rec. Trav. Chim. Pays-Bas. 1988, 107, 15-16.

25. Syrbu S.A., Semeikin A.S. Zh. Org. Khim. 1999, 35, 12621265 (in Russ.).

26. Semeikin A.S., Koifman O.I., Berezin B.D. Khim. Geterotsikl. Soedin. 1986, 798-801 (in Russ.) [Chem. Heterocycl. Compd. 1986, 629-632 (engl. transl.)].

27. Semeikin A.S., Koifman O.I., Berezin B.D., Syrbu S.A. Khim. Geterotsikl. Soedin. 1983, 1359-1362 (in Russ.) [Chem. Heterocycl. Compd. 1983, 1082-1083 (engl. transl.)]

28. Mikhalitsyna E.A., Tyurin V.S., Nefedov S.E., Syrbu S.A., Semeikin A.S., Koifman O.I., Beletskaya I.P. Eur. J. Inorg. Chem. 2012, 5979-5990.

29. Kolodina E.A., Lubimova T.V., Syrbu S.A., Semeikin A.S. Macroheterocycles 2009, 2, 33-41.

30. Kolodina E.A., Syrbu S.A., Semeikin A.S., Koifman O.I. Zh. Org. Khim. 2010, 46, 136-141 (in Russ.). 